Case report

\title{
Disseminated cysticercosis and role of spin echo short tau inversion recovery sequence imaging: Case based review
}

\author{
Lakshmi Narasimhan Ranganathan $^{\mathrm{a}, *}$, Venkatraman Karthikeayan ${ }^{\mathrm{a}}$, \\ Arun Shivaraman $\mathrm{MM}^{\mathrm{a}}$, Guhan $\mathrm{R}^{\mathrm{a}}$, Venkateswaran $\mathrm{KJ}^{\mathrm{a}}$, Thamil Pavai $\mathrm{N}^{\mathrm{a}}$, \\ Manmohan Mehndiratta ${ }^{\mathrm{b}}$ \\ a Institute of Neurology, Madras Medical College, Chennai, India \\ b Janakpuri Super Specialty Hospital, Delhi, India
}

\section{A R T I C L E I N F O}

\section{Article history:}

Received 24 May 2017

Accepted 12 October 2017

Available online 28 October 2017

\section{Introduction}

Disseminated Cysticercosis is a systemic parasitic infection by the larval stage of the pork tape worm Taenia solium. It presents predominantly with neurological and extra neurological symptoms. Neurocysticercosis is involvement of the central nervous system by the encysted larval stage, 'cysticercus cellulosae' of the pork tapeworm Taenia solium. Neurocysticercosis is widely prevalent in the tropic. It is one of the commonest causes of adult onset seizure in tropics. In this report we discuss two cases of disseminated cysticercosis and the current concepts in the imaging and management of disseminated cysticercosis.

\section{Case Vignette 1}

60 year old male, a Chef and marriage contractor by occupation presented with change in personality and behaviour for 1 year duration. He had low grade intermittent fever one year ago which responded to antipyretics. Over the next 3 months he had multiple episodes of generalised tonic clonic seizures. Each episode lasted for 2 min duration with post ictal confusion. He had symptoms and signs executive dysfunction, impairment in attention, calculation and memory. He had emotional incontinence with spells of anger and social withdrawal. Deficits were noted in visuospatial domain. He subsequently developed bradykinesia with hypophonia and unconcerned micturition. Substance abuse history was positive for alcohol consumption and smoking. Examination had also revealed

\footnotetext{
* Corresponding author at: Institute of Neurology, Madras Medical College, Park Town, Chennai 600003, India.

E-mail address: Inmayura@yahoo.com (L.N. Ranganathan).
}

constructional apraxia, ideational and ideomotor apraxia. Perseveration was observed with presence of frontal release signs. Oculomotor examination revealed slow saccades and broken pursuit. He was able to move his limbs to commands. Reflexes were preserved. Multiple well circumscribed non-tender nodules were palpable beneath the skin and also in the muscles all over the body. His blood investigations including complete blood count, urea, creatinine, liver enzymes, electrolytes, glucose were normal. Magnetic Resonance Imaging of whole body revealed multiple tiny ring enhancing lesion scattered throughout the brain (starry sky pattern) with nodular lesions of varying stages and size seen throughout the body including eyelids, neck, shoulder, thigh, gluteal musculature (Image 1). A diagnosis of disseminated cysticercosis was made. He was treated with steroids and phenytoin. His cognitive functions improved in the domains of attention span and recent memory. During his hospital stay he had no episodes of loss of consciousness or seizures.

\section{Case Vignette 2}

32 year old male, painter by occupation presented with complaints of recurrent seizures and headache for 2 weeks. His seizure starts with visual auras involving colourful ill formed designs progressing to twitching in right upper and lower limb. Patient becomes unaware of the events and falls down with tonic clonic movements all four limbs. He has involuntary micturition and tongue bite and regains consciousness with confusion $30 \mathrm{~min}$ later. He gives history of headache holo cranial, dull aching, aggravated on lying down and worsened prior to seizure and early morning. He stopped working for two weeks and stayed home. No change in behaviour or personality was noted. He performed his daily activities without difficulty. 5 years ago he had similar 

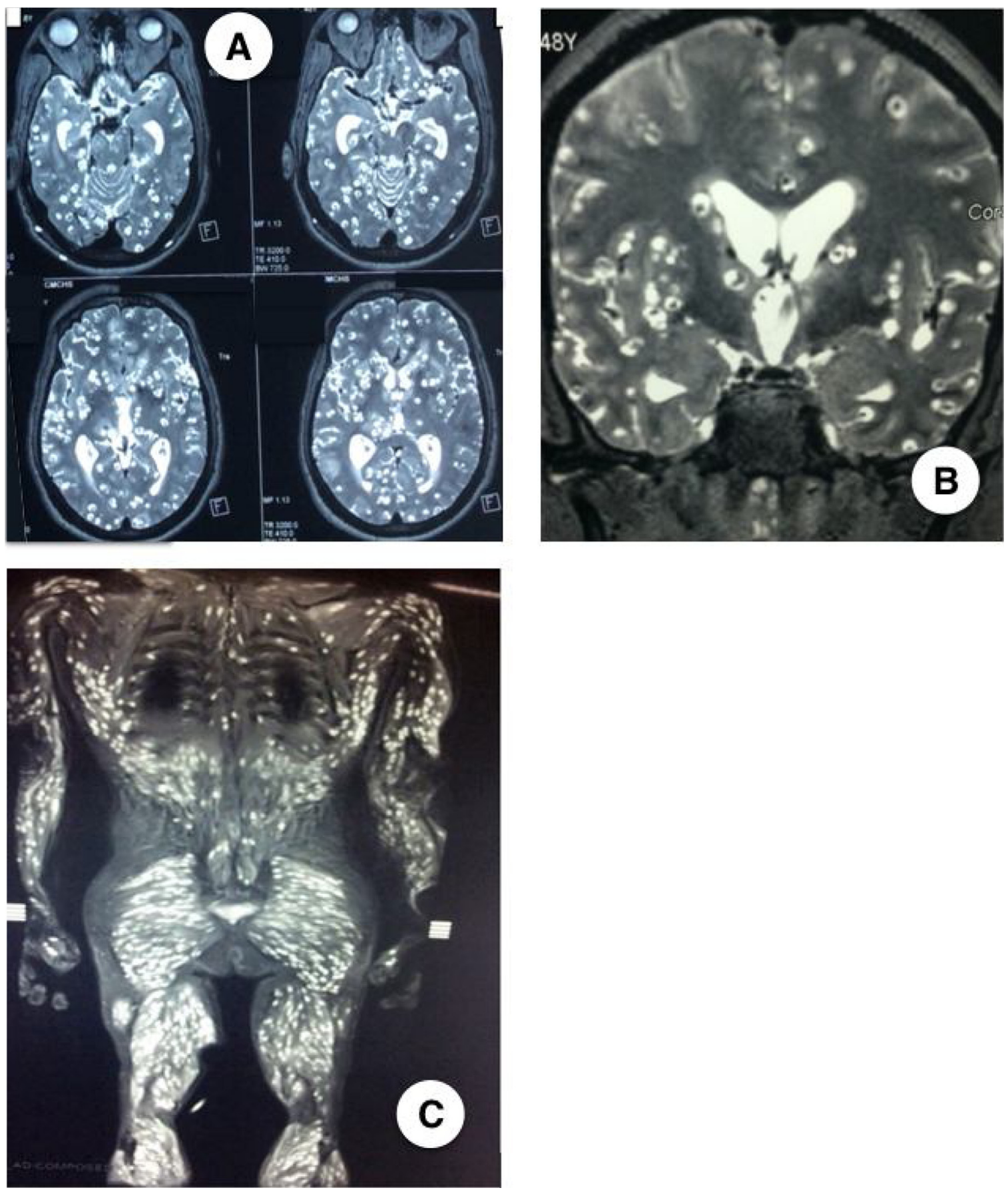

Image 1. Multiple cysts of varying stages seen (starry sky pattern) A, B. Disseminated cysts seen throughout the body in whole body MRI(C).

episode of seizures and was treated with antiepileptics. He remained seizure free for 2 years and was withdrawn of antiepileptics. Examinations revealed normal vitals. He was conscious and attentive. He was oriented but performed poor in calculation and recall of words. His language, constructional ability, praxis, gnosis and executive functions were normal. Blood investigations were within normal limits. Magnetic Resonance imaging of the whole body showed scatted ring enhancing lesions in the cortex with peri lesional edema in the right parieto occipital region. Multiple nodular enhancing lesions were also noted in the neck, thigh, gluteal regions (Image 2). A diagnosis of disseminated cysticercosis was made. Patient was started on steroids and antiepiletics. His headache subsided and throughout the hospital course he did not have any episode of seizure.

\section{Discussion}

Cysticercosis is a systemic infection in humans caused by ingestion of Taenia solium ova. The intestinal infection in humans by the adult tapeworm is known as Taeniasis. If left untreated, the tapeworm can survive inside the body for many years, leading to complications. Cysticercosis develops when the larvae of $T$. solium, following ingestion of the ova invade the body and develop in the muscles, skin and eyes. If larvae invade the central nervous system, 

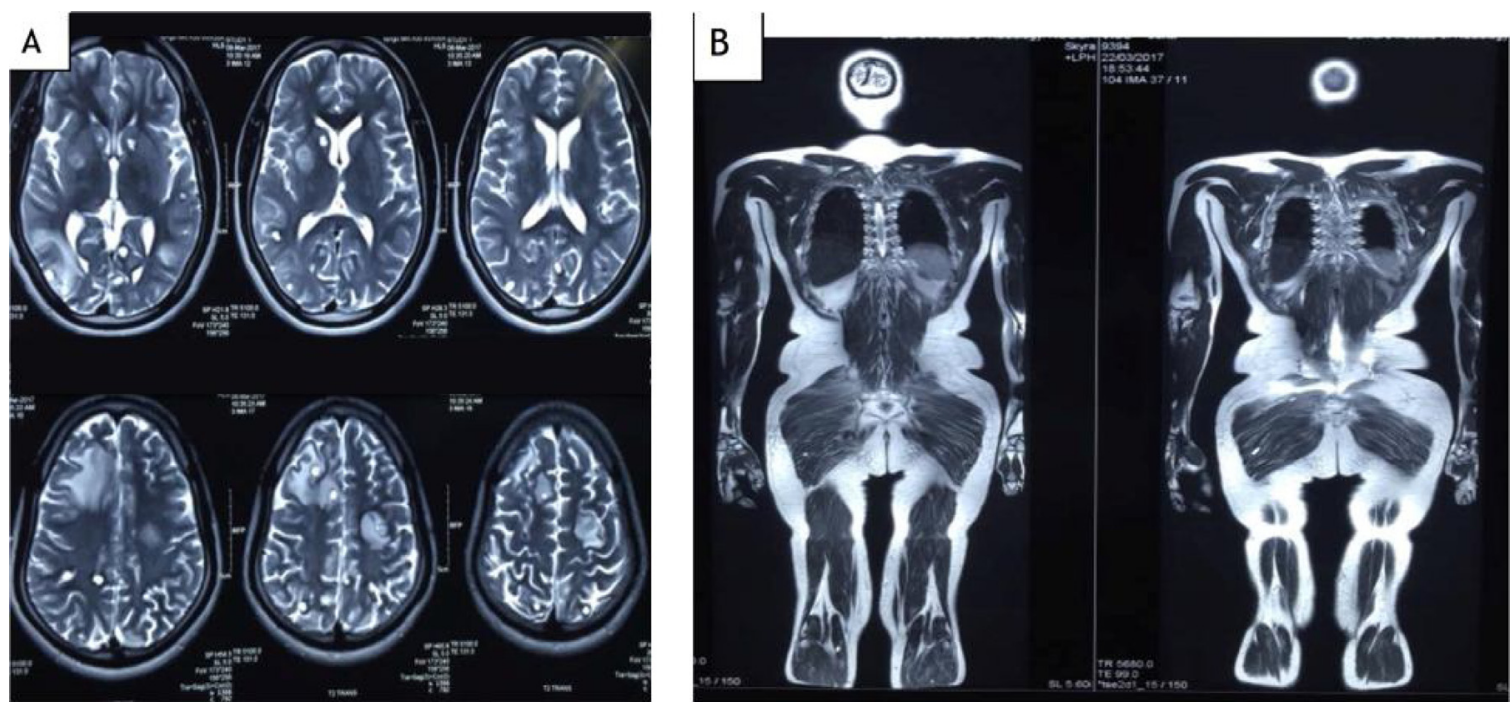

Image 2. Showing multiple cysts in the brain parenchyma with perilesional edema (A), Whole body MRI showing cyst in the gluteal muscle (B).

the infection leads to Neurocysticercosis. Disseminated cysticercosis is characterised by cystic lesions in the brain in combination with atleast one of the following additional sites: skeletal muscle, subcutaneous tissue, intraorbital or the viscera including liver, spleen, heart and lungs. Fulminant neurocysticercosis is characterised by involvement of two or more of the following combination: cerebral parenchyma, intraventricular, cisternal, meningeal (brain and spinal cord), spinal cord parenchyma with or without involvement of eye (Image 3). ${ }^{1,2}$

About 50 million people are affected by epilepsy and more than $80 \%$ of them live in developing countries. For decades latin America had a serious problem with cysticercosis. The disease is endemic in
South-East Asia and is emerging in parts of sub-saharan Africa. Incidence of disease in India is unknown. Mahajan reported that $2.3 \%$ of the general population in and around Chandigarh, had antibody titers positive for Cysticercosis. Seropositivity was greater in rural areas and amongst the socioeconomically deprived people with unhygienic habits. ${ }^{3}$

Human Cysticercosis is an important cause of epilepsy and neurological morbidity in many developing countries. Cysts occur especially in striated muscles, subcutaneous tissues, the nervous system and the eye. Cysticercosis becomes symptomatic in the nervous system, the eye, musculoskeletal system (Image 3 ). Neurocysticercosis is a pleomorphic disease whose clinical

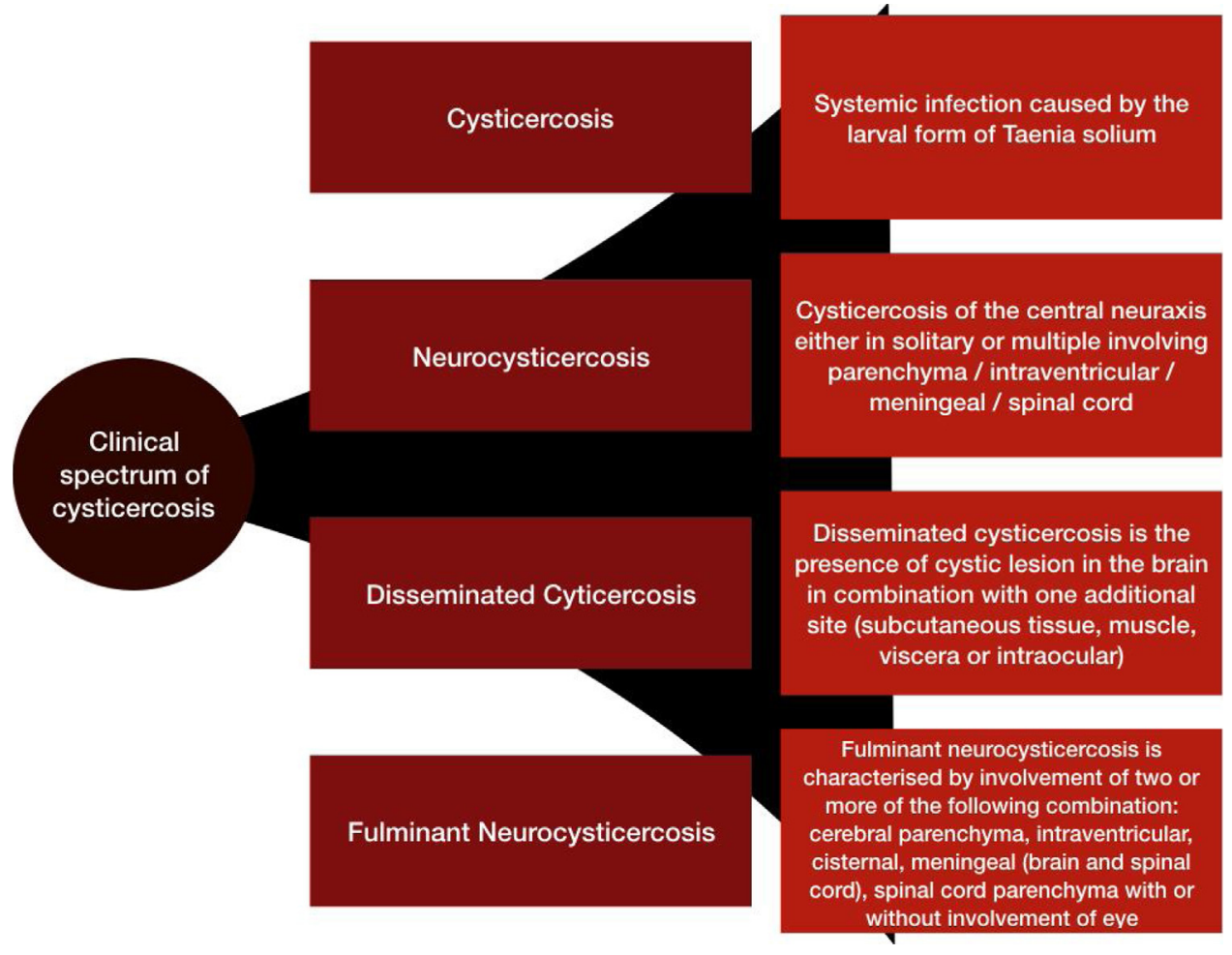

Image 3. Clinical spectrum of cysticercosis. 
manifestations vary with the number, size, location and stage of cysticerci as well as the intensity of the host's immune response.

Neurocysticercosis is caused by the encysted larval stage, 'cysticercus cellulosae' of the pork tapeworm Taenia solium. The parenchymal cysts may remain dormant for many years, and symptoms (e.g. seizures) usually coincide with larval death and subsequent intense inflammatory reaction induced by larval antigens. Subsequently, the cyst transforms into a granuloma. The cyst then shrinks and granuloma eventually calcify or more frequently disappear completely. ${ }^{4}$ This clinico pathological pattern of spontaneous resolution or calcification has important implications for diagnosis. Late-onset seizures in otherwise healthy individuals in endemic areas are highly suggestive of Neurocysticercosis. Common manifestations include seizures, focal neurological signs, intracranial hypertension, cognitive decline, cerebellar ataxia, symptoms of hydrocephalus and psychiatric disorders. The major forms of Neurocysticercosis are parenchymal, ventricular, subarachnoid, spinal and orbital. Ventricular and basal cisternal locations are considered to be malignant forms owing to the intense inflammation associated with the lesion. It is associated with mortality rate of $50 \%$ when hydrocephalus is present (Image 4)..$^{5}$

Natural evolution of Neurocysticercosis takes two forms. Encephalitic stage is characterised by marked cerebral edema, raised intracranial tension, altered mentation and also progressive cognitive decline. Non encephalitic stage is characterised by noninflamed cysts in brain and presents as seizure. Genetic factors play a role in the transformation of massive Neurocysticercosis into encephalitic form.

The diagnostic criteria for Neurocysticercosis proposed in 2001 (Table 1 ) has been widely accepted by medical fraternity. ${ }^{6}$
Imaging modalities utilised in diagnosing neurocysticercosis include CT and MRI brain. In general MRI is considered to produce better results than CT brain. High-resolution ultrasound is useful in demonstrating subretinal cysticercosis. Neurocysticercosis lesions are found in parenchyma of brain, subarachnoid space, ventricles, spinal cord and eye. Brain parenchyma is the most common location in CNS followed by ventricular cysts, subarachnoid space and spinal cord in descending order.

Neurocysticercus lesions pass through four pathological stages (vesicular, colloidal, granular-nodular and calcified). Each stage produces different findings on imaging. Vesicular (active) stage in CT appears as hypodense lesions with scolex within (hole in dot sign). In MRI, it appears as lesions that are isointense with CSF on T1 and T2 imaging. Perilesional edema is absent in this stage. Colloidal cysts appear as contrast enhancing lesion with perilesional edema. Granular cysts present as contrast enhancing lesion in CT brain. In MRI they present as contrast enhancing lesion with perilesional edema and hyperintense rim around the edematous area. Calcified cysts present as hyperdense lesions in CT brain with or without edema or contrast enhancement. Calcified lesion with perilesional edema may be associated with increased risk of seizures. The perilesional edema in calcified cyst is due to inflammatory response against the cysticercal antigens released during micro remodelling of the calcific lesion. Yet another cause for the edema could be changes secondary to the seizures itself. It may be due to release of excitatory neurotransmitter, damage to neurons and disruption of blood brain barrier. ${ }^{7}$

In the course of evolution of the cyst through various stages, it passes from non contrast enhancing to contrast enhancing in contrast imaging. The non contrast enhancement indicates viable cyst with minimal inflammatory host immune response. The

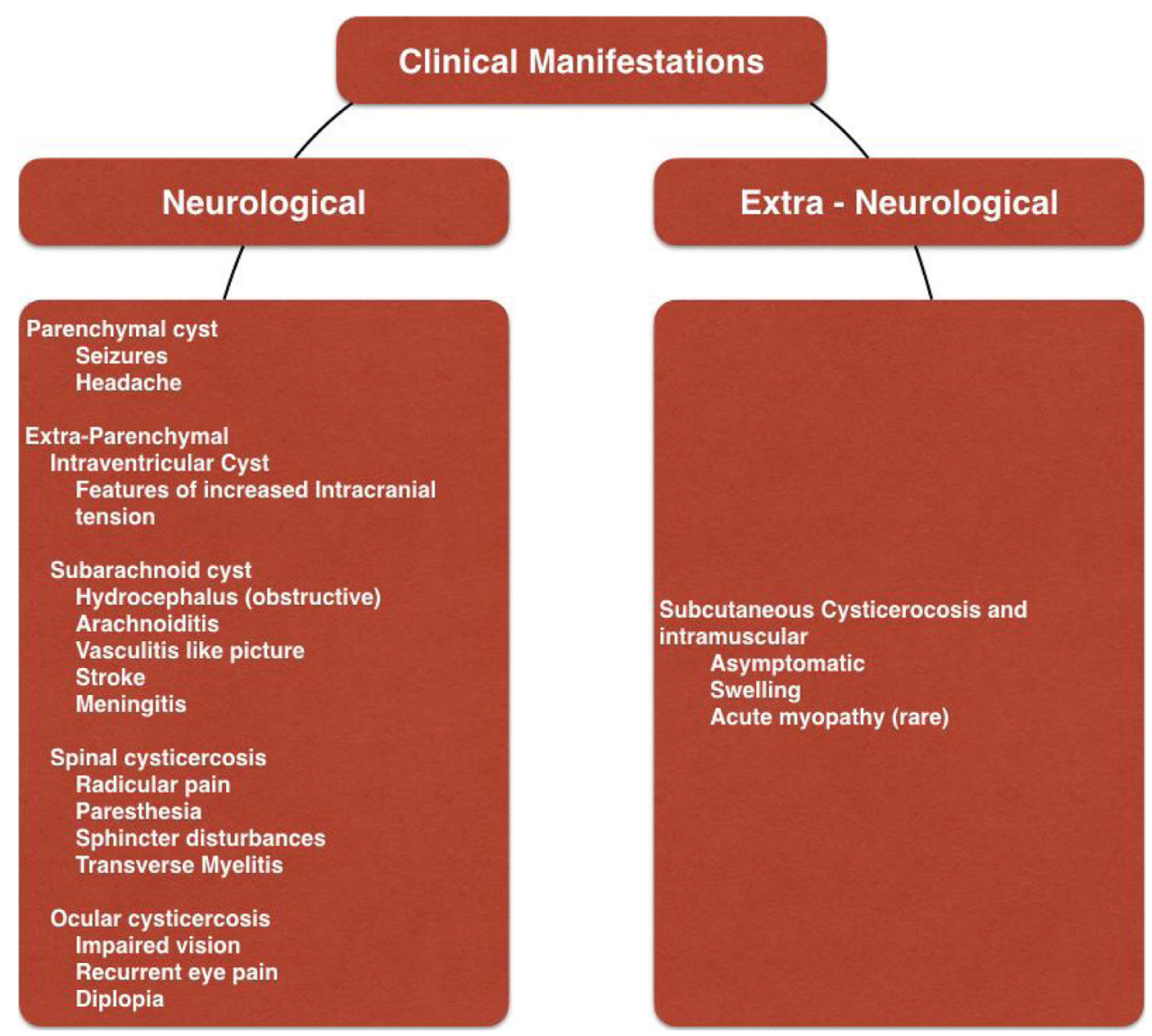

Image 4. Clinical Manifestations of Cysticercosis. 
Table 1

Diagnostic Criteria for Neurocysticercosis. (Del Brutto et al. ${ }^{3}$ ).

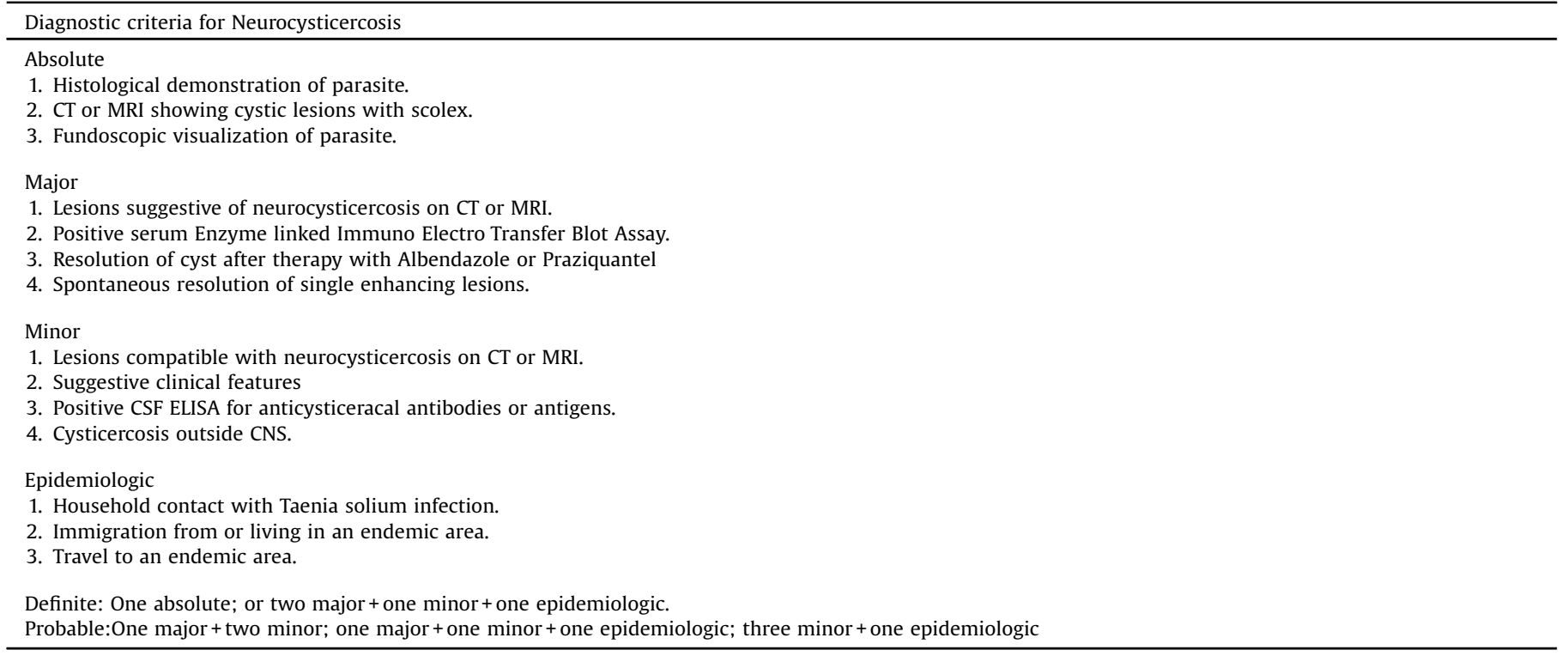

contrast enhancement indicates non viable cyst with inflammatory host immune response. ${ }^{8}$

Neuroimaging in intraventricular Neurocysticercosis and meningeal neurocysticercosis reveals the presence of cystic lesions isointense with CSF in T1 and T2 weighted imaging within the ventricle and the cisternal spaces respectively. The thin wall of the cyst may be demonstrated in T2 sequences and in contrast enhanced sequences. High resolution sequences such as 3D construction interference in steady state (CISS) may help delineate the lesions better. Inflammation adjacent to the ventricles may be demonstrated by contrast enhancement in the ependyma. The most common site of intraventricular involvement includes 4th and 3 rd ventricle. The most common cisternal spaces involved include prepontine, suprasellar and ambient cistern. Conglomeration of lesions and adjacent leptomeningeal inflammation may be seen in meningeal neurocysticercosis. Spinal Neurocysticercosis may be present in the sub arachnoid space or in the intraparenchymal location. Intraorbital cysticercosis may be present in the extra ocular muscles, posterior pole of the eye, vitreous humour and subconjunctival region. ${ }^{9}$

Proton magnetic resonance spectroscopy (pMRS) can also be used along with MRI brain as they differentiate between inflammatory granulomas like neurocysticecosis and tuberculomas depending on the biochemical substance which can be measured. MR spectroscopy showing lipid peaks at 0.9 (corresponding to terminal methyl group), 1.3 (corresponding to methylene group), 2.0, 2.8 (corresponding to fatty acid chain) $\mathrm{ppm}$ in the absence of amino acids and succinate is suggestive of tuberculoma. MR spectroscopy showing threonine peaks at $1.3 \mathrm{ppm}$ and second peak at 3.6 which inverts on long TE sequences, alanine at $1.5,1.6 \mathrm{ppm}$, succinate at $2.4 \mathrm{ppm}$ and glycine, glutamate and lactate peaks with reduction in NAA is suggestive of Neurocysticercosis. The presence of creatinine peak and absence of fumarate and malate in cysticercal cyst helps to differentiate from echinococcal cyst in which malate and fumarate are present and creatinine is absent. The creatinine in cysticercal cyst is derived from the smooth muscles present in the wall of the cyst and by diffusion into the fluid from the surrounding tissue. ${ }^{10,11}$

Magnetic resonance imaging with fast spin-echo short time inversion-recovery (STIR) is a new technique that allows whole body imaging in a reasonable time. The advantage of STIR imaging is the absence of radiation exposure. STIR imaging is useful in assessing the presence and the extent of metastasis in cancer patients, assessing diseases like polymyositis and infections like neurocysticercosis and other systemic infections. ${ }^{12}$

STIR sequence image consists of combined T1, T2 and proton density imaging. Most pathological tissues are rich in protons resulting in high signal intensity on STIR imaging. STIR imaging also produces fat suppression based on $\mathrm{T} 1$ relaxation time between fat and other tissues. Fluid (pleural effusion), edema, cerebrospinal fluid has very high signal intensity. Tumors and gray matter of brain produces high intensity. Muscle, white matter have intermediate signal whereas subcutaneous tissue, fat and cortical bone produces low signal intensity. ${ }^{13}$

Whole body STIR imaging can be used as a screening tool for assessing disseminated neurocysticercosis. Neurocysticercosis lesions appear as hyperintense lesions on STIR imaging. Initiation of anihelminthic drugs without immunosuppressive agents in the presence of disseminated neurocysticercosis can result in fatal reactions. ${ }^{14}$ STIR imaging can also be useful in assessing the response to treatment with anti helminth drugs.

Treatment of disseminated cysticercosis has been controversial and poorly supported by evidence. Antihelminthics, corticosteroids and anti epileptic drugs play a role in the treatment of neurocysticercosis.

Anti-helminthic drugs namely albendazole and praziquantel have been tried with varying success. Albendazole has shown superior efficacy in treating isolated brain cystic lesions and have no role in calcified cysts.

Studies have shown that albendazole in comparison with placebo or no treatment, when used in patients with disseminated neurocysticercosis, had increased risk of seizure recurrence, increased hospital admissions in the initial year, higher symptom risk in the initial 3 months, no effect on the radiological resolution in the initial year and increased headache in the absence of combination treatment with corticosteroids. ${ }^{15,16}$ However, in patients with viable lesions of upto 20 lesions, the use of albendazole results in positive effects of decreasing the seizures and number of lesions. ${ }^{17}$ The use of praziquantel in the treatment of neurocysticercosis resulted in the lack of resolution of 
symptoms at three months, radiological reduction of lesions at three months and statistically insignificant increase in adverse events during treatment. The use of albendazole in comparison with praziquantel in the treatment of viable neurocysticercosis resulted in improved resolution clinically and radiologically at three months without any significant difference in the recurrence of seizures and adverse events between the two groups. The combination therapy of albendazole with praziquantel in comparison with mono therapy alone resulted in increased reduction of lesions radiologically, however was not associated with change in seizure frequency or adverse events. The use of albendazole in combination with steroids in comparison with monotherapy did not result in change in seizure frequency or radiological resolution. However in combination with corticosteroids was associated with reduction in the occurrence of headache. Treatment with albendazole for shorter duration ( $<8$ days) in comparison with longer duration ( $>8$ days) for disseminated neurocysticercosis was not associated with change in seizure frequency or radiological resolution. However it was associated with fewer adverse events in those with shorter duration of treatment. The use of anti helminthic therapy in patients with disseminated neurocysticercosis requires a larger evidence base. ${ }^{16,18-27}$

Studies have shown the efficacy of antiepileptic drugs (AEDs), phenytoin and carbamazepine, in the treatment of neurocysticercosis. Newer anti epileptic drugs, Levetiracetam and topiramate are as efficacious as the older drugs. AEDs are initiated in patients presenting with seizures and in high risk patients. Factors contributing to high risk include multiple lesions with increasing inflammation as evidenced by contrast enhancement. The duration of anti epileptic therapy is inconclusive. In patients with multiple lesions and refractory seizure, AEDs would be required indefinitely. Tapering of AEDs may be attempted six to twelve months following radiological resolution of lesions. ${ }^{28-33}$

Corticosteroids administered in patients receiving anti helminthic therapy in parenchymal neurocysticercosis is associated with reduced seizure recurrence and radiological improvement. Steroids are the drug of choice in cysticercal encephalitis with diffuse cerebral edema. Adminstration of steroids in subarachnoid neurocysticercosis results in reduction of symptoms. ${ }^{34-38}$

Surgical removal of cyst with venticulo-peritoneal shunting is advised when the cyst causes hydrocephalus due to occlusion of the ventricular system.

We observed two different forms of Neurocysticercosis in our patients. First patient presented with the encephalitic form and the second patient in the non-encephalitic form. The use of whole body STIR imaging was instrumental in diagnosing the disseminated cysticercosis in both the patients. We have treated both the patients with steroids and antiepileptics. Anti helminths were avoided in the above patients due to disseminated nature and increased risk of clinical worsening. Both our patients are in follow up and are symptomatically better. The natural course of the cysticercosis is the passage of the larvae into cyst form followed by human defence against them leading to death of these cysts and calcification. This has raised the question of starting antihelminthic drugs in disseminated cysticercosis. Consensus statement on using antihelminthic drugs in disseminated cysticercosis is currently not available. Until then two schools of thoughts will prevail as to use of antihelminthic drugs in disseminated cysticercosis.

\section{Conclusion}

Any adult onset seizure especially in tropics should lead to suspicion of this condition. Neurocysticercosis should also be considered in patients with rapidly progressive dementia as it is potentially reversible by treatment. Use of whole body MR imaging with Short Tau Inversion Recovery (STIR) should be planned in every Neurocysticercosis to identify dissemination. Controversy exists regarding the use of antihelminthics in disseminated cysticercosis which requires further studies and a wider literature base.

\section{Conflict of interest}

None.

\section{References}

1. Garg RK. Diagnostic criteria for neurocysticercosis: some modifications are needed for Indian patients. Neurol Ind. 2004;52:171-177.

2. Jawale R, Duberkar D. Disseminated cysticercosis. Neurology. 2015;84(January (3)):327.

3. Mahajan RC. Geographical distribution of human cysticercosis. In: Flisser A, Willms K, Laclette TP, Larralde C, Ridaura C, NBeltram F, eds. Cysticercosis: Present State of Knowledge and Perspective. New York: Academic Press; 1982:39-46.

4. Chopra JS, Kaur U, Mahajan RC. Cysticercosis and epilepsy: a clinicaland serological study. Trans R Soc Trop Med Hyg. 1981;75:518-520.

5. Wadia Noshir, Desai Shrinivas, Bhatt Mohit. Disseminated cysticercosis. Brain. 1988;111(3):597-614.

6. Del Brutto OH, Rajshekhar V, White Jr. ACJr., et al. Proposed diagnostic criteria for neurocysticercosis. Neurology. 2001;57:177-183.

7. Nash Theodore E, Patronas Nicholas J. Edema associated with calcified lesions in neurocysticercosis. Neurology. 1999;53(4):777.

8. Garcı'a Héctor H, Del Brutto Oscar H. Imaging findings in neurocysticercosis. Acta Trop. 2003;87(1):71-78.

9. Lerner A, Shiroishi MS, Zee CS, Law M, Go JL. Imaging of neurocysticercosis. Neuroimaging Clin N Am. 2012;22(November (4)):659-676.

10. Pandit S, Lin A, Gahbauer H, et al. MR spectroscopy in neurocysticercosis. J Comput Assist Tomogr. 2001;25(6):950-952.

11. Chavhan Govind B, Babyn Paul S. Whole-body MR imaging in children: principles, technique, current applications, and future directions. Radiographics. 2011;31(6):1757-1772.

12. Garg M, Chawla S, Prasad KN, et al. Differentiation of hydatid cyst from cysticercus cyst by proton MR spectroscopy. NMR Biomed. 2002;15(5):320326.

13. Kellenberger CJ, Epelman M, Miller SF, et al. Fast STIR whole-body MR imaging in children 1. Radiographics. 2004;24(5):1317-1330.

14. Udare A, Juvekar S, Medhi S, et al. Disseminated cysticercosis: role of whole body Magnetic Resonance Imaging. Ann Parasitol. 2016;62(2):149.

15. Garcia HH, Del Brutto OH. Taenia solium cysticercosis. Infect Dis Clin North Am. 2000;14(March (1)):97-119.

16. Abba K, Ramaratnam S, Ranganathan LN. Anthelmintics for people with neurocysticercosis. Cochrane Database Syst Rev. 2010;(Jan (3)).

17. Garcia HH, Pretell EJ, Gilman RH, et al. Cysticercosis Working Group in PeruA trial of antiparasitic treatment to reduce the rate of seizures due to cerebral cysticercosis. N Engl J Med. 2004;350:249-258.

18. Alarcon F, Escalante L, Duenas G, Montalvo M, Roman M. Neurocysticercosis: short course of treatment with albendazole. Arch Neurol. 1989;46(11):12311236.

19. Alarcon F, Duenas G, Diaz M, Cevallos N, Estrada G. Short course of albendazole therapy for neurocysticercosis: a prospective randomized trial comparing three days, eight days and the control group without albendazole. Rev Ecuat Neurol. 2001;10:1-2.

20. Carpio A, Kelvin EA, Bagiella E, et al. Effects of albendazole treatment on neurocysticercosis: a randomised controlled trial. J Neurol Neurosurg Psychiatry. 2008;79:1050-1055.

21. Cruz I, Cruz ME, Carrasco F, Horton J. Neurocysticercosis: optimal dose treatment with albendazole. J Neurol Sci. 1995;133(1-2):152-154.

22. Del Brutto OH, Campos X, Sanchez J, Mosquera A. Single-day praziquantel versus 1-week albendazole for neurocysticercosis. Neurology. 1999;23 (52):1079-1081.

23. Garcia H, Gilman R, Catacora M, et al. Serologic evolution of neurocysticercosis patients after antiparasitic therapy. J Infect Dis. 1997;175:486-489.

24. Garcia HH, Pretell EJ, Gilman RH, et al. A trial of antiparasitic treatment to reduce the rate of seizures due to cerebral cysticercosis. N Engl J Med. 2004;350 (3):249-258.

25. Singhi P, Jain V, Khandelwal N. Corticosteroids versus albendazole for treatment of single small enhancing computed tomographic lesions in children with neurocysticercosis. I Child Neurol. 2004:19(5):323-327.

26. Sotelo J, Escobedo F, Penagos P. Albendazole vs praziquantel for therapy for neurocysticercosis: a controlled trial. Arch Neurol. 1988;45(5):532-534.

27. Sotelo J, Del Brutto OH, Penagos P, et al. Comparison of therapeutic regimen of anticystercal drugs for parenchymal brain cysticercosis. J Neurol. 1990;237:6972 .

28. Garcia HH, Pretell EJ, Gilman RH, et al. A trial of antiparasitic treatment to reduce the rate of seizures due to cerebral cysticercosis. $N$ Engl J Med. 2004;350:249. 
29. Del Brutto OH. Prognostic factors for seizure recurrence after withdrawal of antiepileptic drugs in patients with neurocysticercosis. Neurology. 1994; $44: 1706$

30. Carpio A, Hauser WA. Prognosis for seizure recurrence in patients with newly diagnosed neurocysticercosis. Neurology. 2002;59:1730.

31. Rajshekhar V, Jeyaseelan L. Seizure outcome in patients with a solitary cerebral cysticercus granuloma. Neurology. 2004;62:2236.

32. Verma A, Misra S. Outcome of short-term antiepileptic treatment in patients with solitary cerebral cysticercus granuloma. Acta Neurol Scand. 2006;113:174.

33. Nash TE, Pretell EJ, Lescano AG, et al. Perilesional brain oedema and seizure activity in patients with calcified neurocysticercosis: a prospective cohort and nested case-control study. Lancet Neurol. 2008;7:1099.

34. Mall RK, Agarwal A, Garg RK, et al. Short course of prednisolone in Indian patients with solitary cysticercus granuloma and new-onset seizures. Epilepsia. 2003;44:1397.
35. Nash TE, Singh G, White AC, et al. Treatment of neurocysticercosis: current status and future research needs. Neurology. 2006;67:1120.

36. Prakash S, Garg RK, Kar AM, et al. Intravenous methyl prednisolone in patients with solitary cysticercus granuloma: a random evaluation. Seizure. 2006;15:328.

37. Garg RK, Potluri N, Kar AM, et al. Short course of prednisolone in patients with solitary cysticercus granuloma: a double blind placebo controlled study. J Infect. 2006;53:65.

38. Kishore D, Misra S. Short course of oral prednisolone on disappearance of lesion and seizure recurrence in patients of solitary cysticercal granuloma with single small enhancing CT lesion: an open label randomized prospective study. J Assoc Physicians India. 2007;55:419. 